Methodology article

Open Access

\title{
Evaluation of high efficiency gene knockout strategies for Trypanosoma cruzi

\author{
Dan Xu ${ }^{\dagger 1}$, Cecilia Pérez Brandán ${ }^{\dagger 2}$, Miguel Ángel Basombrío ${ }^{2}$ and \\ Rick L Tarleton*1
}

Address: ${ }^{1}$ Department of Cellular Biology and Center for Tropical and Emerging Global Diseases, University of Georgia, Athens, GA 30602, USA and ${ }^{2}$ Instituto de Patologia Experimental, Universidad de Salta, 4400 Salta, Argentina

Email: DanXu - dxu@uga.edu; Cecilia Pérez Brandán - perezbrandan@yahoo.com.ar; Miguel Ángel Basombrío - basombri@unsa.edu.ar; Rick L Tarleton* - tarleton@cb.uga.edu

* Corresponding author †Equal contributors

Published: II May 2009

BMC Microbiology 2009, 9:90 doi:10.1 186//47|-2180-9-90
Received: 18 December 2008

Accepted: II May 2009

This article is available from: http://www.biomedcentral.com/147I-2180/9/90

(C) 2009 Xu et al; licensee BioMed Central Ltd.

This is an Open Access article distributed under the terms of the Creative Commons Attribution License (http://creativecommons.org/licenses/by/2.0), which permits unrestricted use, distribution, and reproduction in any medium, provided the original work is properly cited.

\begin{abstract}
Background: Trypanosoma cruzi, a kinetoplastid protozoan parasite that causes Chagas disease, infects approximately 15 million people in Central and South America. In contrast to the substantial in silico studies of the T. cruzi genome, transcriptome, and proteome, only a few genes have been experimentally characterized and validated, mainly due to the lack of facile methods for gene manipulation needed for reverse genetic studies. Current strategies for gene disruption in $T$. cruzi are tedious and time consuming. In this study we have compared the conventional multi-step cloning technique with two knockout strategies that have been proven to work in other organisms, one-step-PCR- and Multisite Gateway-based systems.
\end{abstract}

Results: While the one-step-PCR strategy was found to be the fastest method for production of knockout constructs, it does not efficiently target genes of interest using gene-specific sequences of less than 80 nucleotides. Alternatively, the Multisite Gateway based approach is less timeconsuming than conventional methods and is able to efficiently and reproducibly delete target genes.

Conclusion: Using the Multisite Gateway strategy, we have rapidly produced constructs that successfully produce specific gene deletions in epimastigotes of $T$. cruzi. This methodology should greatly facilitate reverse genetic studies in $T$. cruzi.

\section{Background}

Trypanosoma cruzi is a protozoan parasite and the etiological agent of Chagas disease in humans, also known as American trypanosomiasis. T. cruzi infects over 100 species of mammalian hosts and is the leading cause of infection-induced heart failure in Latin America [1,2]. In 2006, approximately 12,500 deaths have been reported as a result of the clinical complications of $T$. cruzi-induced heart disease and the lack of effective treatment [3].

T. cruzi has four morphologically and physiologically distinct stages. The bloodstream trypomastigotes and intracellular amastigotes stages of parasites are in the mammalian host, whereas epimastigotes and metacyclic 
trypomastigotes develop in the insect vector [4]. The diploid genome of $T$. cruzi contains approximately 40 chromosomes encoding a predicted set of 22,570 proteins, of which at least 12,570 represent allelic pairs [5]. Allelic copies of genes in the hybrid CL Brener genome may vary in sequence by as much as $1.5 \%$, and trisomy has also been suggested in the case of some chromosomes [6,7]. Putative functions could be assigned to $50.8 \%$ of the predicted protein-coding genes on the basis of significant similarity to previously characterized proteins or known functional domains [5].

In contrast to the substantial in silico studies of the T. cruzi genome, only 10 genes have been experimentally characterized by reverse genetics in T. cruzi [8-18]. These genes were all disrupted through homologous recombination, using a DNA cassette that has a drug selectable marker flanked by the coding sequence or the untranslated regions (UTRs) of the target gene. Although effective, this conventional gene knockout approach not only requires identification of multiple compatible restriction sites for ligation reactions and for vector linearization, it also involves multiple restriction digestions, ligations and cloning steps that make the process cumbersome and time-consuming [19]. Given that RNA interference has, to date, failed to function in T. cruzi [20] (in contrast to the situation in the African trypanosomes [21]), a simplified strategy to knockout genes in $T$. cruzi would vastly improve the characterization of the multitude of genes encoding proteins without confirmed or even putative functions.

In this study, we sought to develop a simpler method for the deletion of T. cruzi genes. We compared the conventional multi-step knockout technique with two knockout strategies that have been proven to work in other organisms, one-step-PCR- and Multisite Gateway (MS/GW) based systems. We attempted to knockout the dihydrofolate reductase-thymidylate synthase $(d h f r-t s)$ using all three techniques, and enoyl-CoA hydratase (ech) genes using the two alternative approaches. Our results show that gene-specific sequences of 78 nucleotides used in one-step-PCR strategy are not sufficient to guarantee homologous recombination in T. cruzi. However, the MS/ GW-based approach is able to efficiently disrupt target genes. In addition, using the MS/GW strategy, generation of knockout constructs can be completed in as few as 5 days. The results of this study will provide a powerful new tool for reverse genetic studies of T. cruzi.

\section{Results \\ dhfr-ts gene is disrupted using a conventional $\mathrm{KO}$ construct}

The $d h f r$-ts gene is annotated as two identical alleles in the diploid CL Brener reference strain and codes for dihydro- folate reductase thymidylate syntase [5]. In most organisms these two enzyme activities are present on separate monofunctional enzymes. In contrast, in T. cruzi both enzymes are on the same polypeptide chain, with the DHFR domain at the amino terminus and the TS domain at the carboxy terminus $[22,23]$. Since these enzymes catalyze consecutive reactions in the de novo synthesis of 2'deoxythymidylate (dTMP), they have been used as targets for chemotherapy, as inhibition of either enzyme disrupts the dTMP cycle and results in thymidine auxotrophy [2426].

G418 (geneticin)-resistant parasites were obtained after transfection of the recombination fragment excised from the plasmid pBSdh1f8Neo (Additional file 1: Figure S1) into the Tulahuen strain of T. cruzi. We included a $280 \mathrm{bp}$ $1 \mathrm{~F} 8$ fraction in the construct so as to provide a trans-splicing acceptor site and a putative polyadenylation signal to the drug resistance gene [27]. Figure $1 \mathrm{~A}$ shows the expected genomic loci of $d h f r-t s$ and $1 f 8 N e o$ in $d h f r-t s^{+/-/}$ $\mathrm{Neo}$ parasites. As expected no amplification of the 1 f8Neo was observed in Tulahuen WT (wild type) parasites as shown by PCR with primers N1-N2 (Figure 1B). PCR using primers in the flanking genes corroborates the correct insertion of $1 f 8 \mathrm{Neo}$ gene in $d h \mathrm{fr}^{-t \mathrm{~s}^{+/}}$parasite's genome. When using N3-R1, N3-R2 and N3-R3 combinations, bands of $1.9,2.2$ and $2.65 \mathrm{~kb}$ respectively, were observed, providing further confirmation that the neomycin phosphotransferase gene (Neo) had been inserted in the correct locus (Figure 1C). The insertion in the dhfr-ts locus was also confirmed by Southern Blot analysis with gDNA from cloned $d h f r-t s^{+/-}$and WT parasites digested with Sall and probed with $d h f r$-ts (Figure 1D). When digested with enzymes SalI and probed with dhfr-ts CDS we observe a band of $3.2 \mathrm{~kb}$ in wild type parasites while mutants have a 1092 bp insertion corresponding to the 1 f 8 Neo cassette interrupting the dhfr-ts CDS, resulting in an extra $4.4 \mathrm{~kb}$ band in the mutants.

\section{dhfr-ts gene is replaced using a MS/GW construct}

Since we were able to obtain $d h f r-t s^{+/}$parasites we concluded that this gene would be a good candidate to evaluate the one-step-PCR and Multisite Gateway-based systems for gene knockout constructs in T. cruzi. In the MS/GW recombination fragments, the flanking regions of the gene were used as arms for recombination event, in contrast with the method in Figure 1 where the coding sequence of the gene was used for homologous recombination.

Drug resistant lines produced by the transfection of Tulahuen strain epimastigotes with a recombination fragment obtained from pDEST/dhfr-ts_1F8Hyg plasmid (Additional file 2: Figure S2) were cloned and analyzed by PCR and Southern Blot. Figure 2A shows the expected genomic 
A
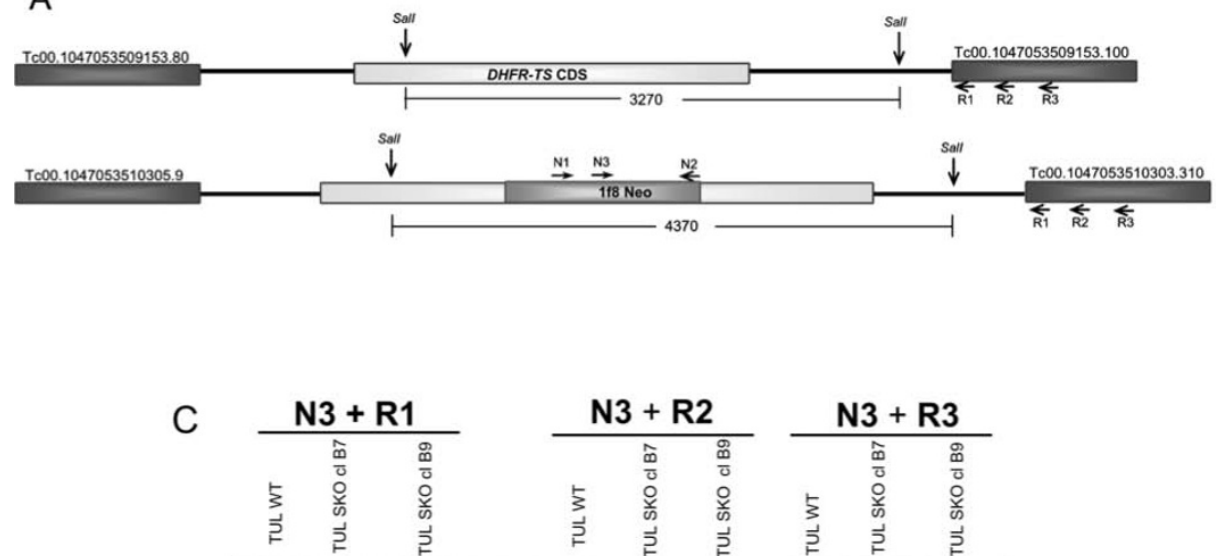

\begin{tabular}{|c|c|c|}
\hline \multicolumn{3}{|c|}{ N3 + R2 } \\
\hline & $\begin{array}{l}\hat{\omega} \\
\stackrel{0}{0} \\
\stackrel{0}{2}\end{array}$ & $\begin{array}{l}\frac{\omega}{0} \\
\circ\end{array}$ \\
\hline$\vec{p}$ & के & 列 \\
\hline
\end{tabular}

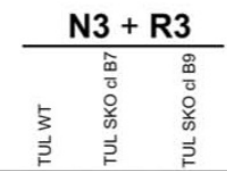

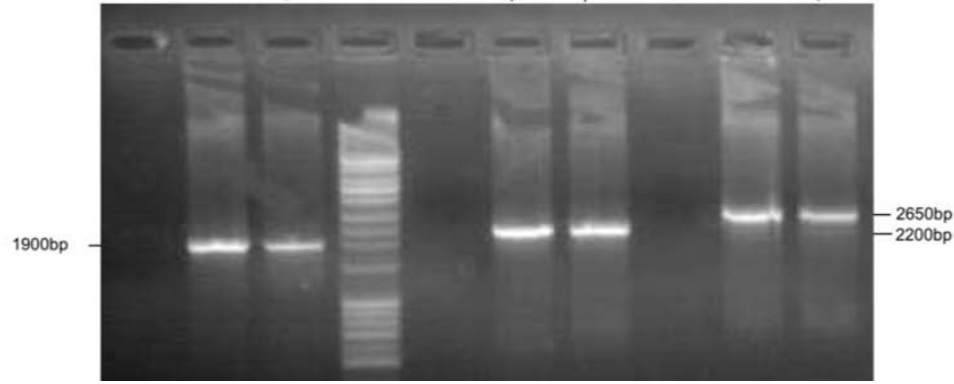

B

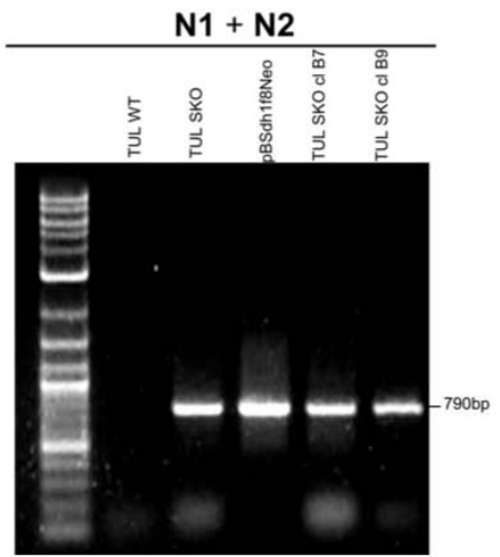

D

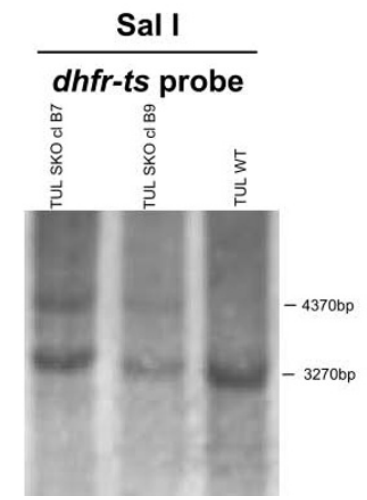

\section{Figure I}

Disruption of dhfr-ts using a conventional KO construct pBSdh I f8Neo. A) Diagram of the expected genomic loci of dhfr-ts and I f8Neo in dhfr-ts $/$-/Neo parasites. B) PCR analysis with Neo specific primers of WT Tulahuen and both uncloned and selected clones of $\mathrm{dhfr}_{\mathrm{fs}} \mathrm{s}^{+/} / \mathrm{Neo}$ parasites. C) PCR analysis with gDNA from selected clones of $\mathrm{dhfr}$-ts ${ }^{+/ / / N e o}$ and WT Tulahuen parasites confirming the expected gene disruption of one allele of the dhfr-ts gene by If8Neo. D) Southern Blot analysis of WT Tulahuen and two $\mathrm{dhfr}$-ts ${ }^{+/} / \mathrm{Neo}$ clones digested with Sall and probed with $\mathrm{dhfr}$-ts probe. Diagram not to scale. Numbers are sizes (bp) of expected products.

loci of $d h f r-t s$ and $1 f 8 H y g$ in the genome of $d h f r-t s^{+/-} / H y g$ parasites; the results of PCR analysis (Figure 2B) confirm the correct insertion of $1 f 8 H y g$ replacing one allele of the dhfr-ts gene (Additional file 3). Southern Blot analysis also showed correct insertion of the 1 f8Hyg cassette replacing one copy of the dhfr-ts gene in the genome. The expected $1312 \mathrm{bp}$ band was observed in BsrGI digested DNA from $d h f r-t s^{+/}$- cloned parasites and probed with Hyg (hygromycin resistance gene) CDS but not in the WT parasites (Figure $2 \mathrm{C}$ ).

\section{Consecutive ech I and ech2 genes are simultaneously} replaced by constructs generated based on MS/GW system T. cruzi ech 1 and $e c h 2$ are tandemly arranged genes (Figure $3 \mathrm{~A})$ with a nucleotide sequence identity of $67 \%$. Both genes encode putative enoyl-CoA hydratase/isomerase $(\mathrm{ECH})$ family proteins, which catalyze the second step in the beta-oxidation pathway of fatty acid metabolism. Analysis of the T. cruzi proteome suggested that enzymes in the fatty acid oxidation pathway, including $\mathrm{ECH}$, are preferentially expressed in amastigotes [28]. Therefore, we hypothesized that we would be able to knockout both ech 1 and ech 2 genes in epimastigotes. The ech locus also provides an opportunity to test whether or not the MS/ GW approach can be used to produce knockouts of multiple genes that are physically linked in the genome.

In T. cruzi, transcript stability and protein translation is largely controlled by 3'UTR and intergenic regions $[29,30]$. The intergenic region of a constitutively expressed gene, gapdh, gives consistently high levels of stable RNA in different constructs and in different life cycle stages [31]. Hence, we included the 3' UTR of gapdh in our constructs, to ensure the expression of the inserted drug resistant 

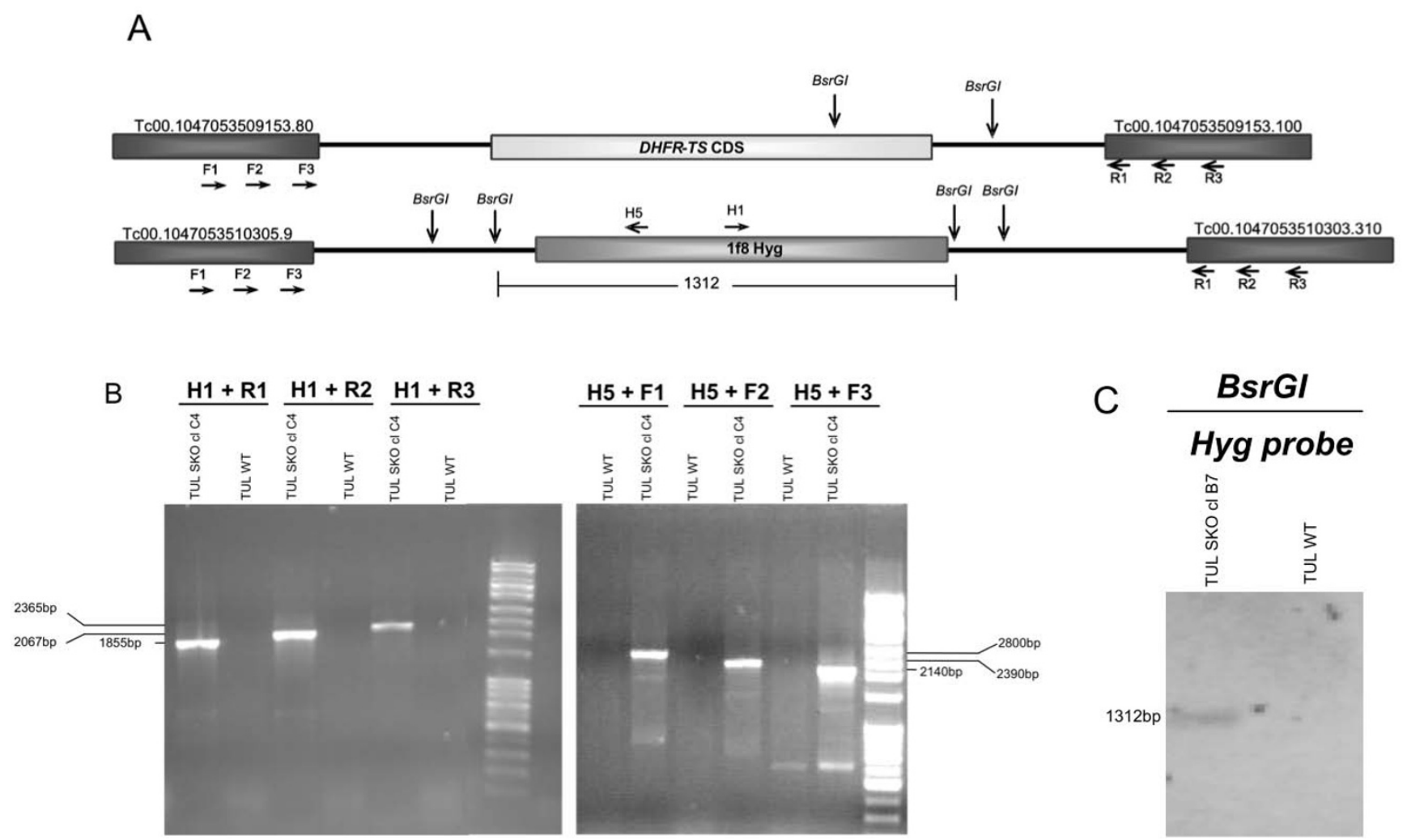

Figure 2

Replacement of dhfr-ts gene with a MS/GW construct pDEST/dhfr-ts_IF8Hyg. A) Schematic of the expected genomic loci of $d h f r-t s$ and I f8Hyg in $d h f r-t s^{+/} / / H y g$ parasites. B) PCR analysis with gDNA from cloned drug resistant parasites and WT Tulahuen parasites confirm the expected gene deletion of one allele of the dhfr-ts gene and correct insertion of If $8 \mathrm{Hyg}$. Primer HI plus the RI, R2 or R3 downstream primers, yield the expected products of I.8, 2.0 and $2.3 \mathrm{~kb}$, respectively and the combination of $\mathrm{H} 5$ plus upstream primers F3, F2 and FI give the predicted bands of 2. I, 2.4 and $2.8 \mathrm{~kb}$ for respectively. See additional file 3: Table S5 for nucleotide sequences of primers. C) Genomic DNA Southern blot analysis of a dhfr-ts ${ }^{+/-/ / H y g}$ Tulahuen clone. gDNA digested with BsrGl and hybridized with labeled Hyg CDS probe. Diagram not to scale. Numbers are sizes (bp) of expected products.

genes in the epimastigote stage. Transfection of the DNA fragment from pDEST/ech-Hyg-GAPDH (Additional file 4: Figure S3A) resulted in parasite lines that were resistant to Hyg selection. Figure 3A shows the expected genomic loci of $e c h$ and $H y g-G A P D H-I R$ in the genome of $e \mathrm{ch}^{+/-} / \mathrm{Hyg}$ parasites. PCR analysis with the genomic DNA from the drug resistant parasites and WT CL confirmed the expected gene replacement of ech 1 and $e c h 2$ genes by $H y g-$ GAPDH-IR (Figure 3B); no products were obtained when using WT CL gDNA as the template with primer combinations $\mathrm{f} 2$ and $\mathrm{D}, \mathrm{f} 2$ and $\mathrm{F}, \mathrm{C}$ and $\mathrm{r} 2$, and $\mathrm{E}$ and $\mathrm{r} 2$, whereas products of the expected sizes, $1759 \mathrm{bp}, 2178 \mathrm{bp}, 2696 \mathrm{bp}$ and $2889 \mathrm{bp}$, respectively, were observed with gDNA from $e c h^{+/-/ H y g}$ as the template. Southern blot analysis of EcoR I digested gDNA using the ech1 gene as a probe (Figure 3A and $3 \mathrm{C}$ right panel) showed a 4880 bp band corresponding to the replaced allelic copy of both ech genes was undetected in ech+/-/Hyg, whereas the $3490 \mathrm{bp}$ and 1365 bp bands corresponding to the second allele were retained. In addition, a 2988 bp band and a 1478 bp band corresponding to the inserted $H y g-G A P D H-I R$ were observed in BanI digested gDNA of only the ech+/-/Hyg, but not that of WT CL (Figure 3A and 3C left panel). Taken together, these results confirmed that one copy of each of the tandem ech1 and ech2 genes was replaced by the MS/ GW Hyg-GAPDH-IR knockout cassette.

Similarly, using linearized DNA from pDEST/ech_NeoGAPDH (Additional file 4: Figure S3B), we generated $e \mathrm{ch}^{+/}$ - Neo parasites with one copy of both ech1 and ech2 gene replaced by Neo-GAPDH-3'UTR knockout cassette (Figure $4 \mathrm{~A})$. This result is confirmed by both PCR amplification (Figure 4B) of gDNA of the drug resistant parasites, as PCR with primer combinations $\mathrm{f} 2$ and $\mathrm{B}$, and $\mathrm{f} 2$ and $\mathrm{H}$ generated 1494 bp and 1949 bp bands respectively only in drug resistant parasites. Southern blot hybridization also 


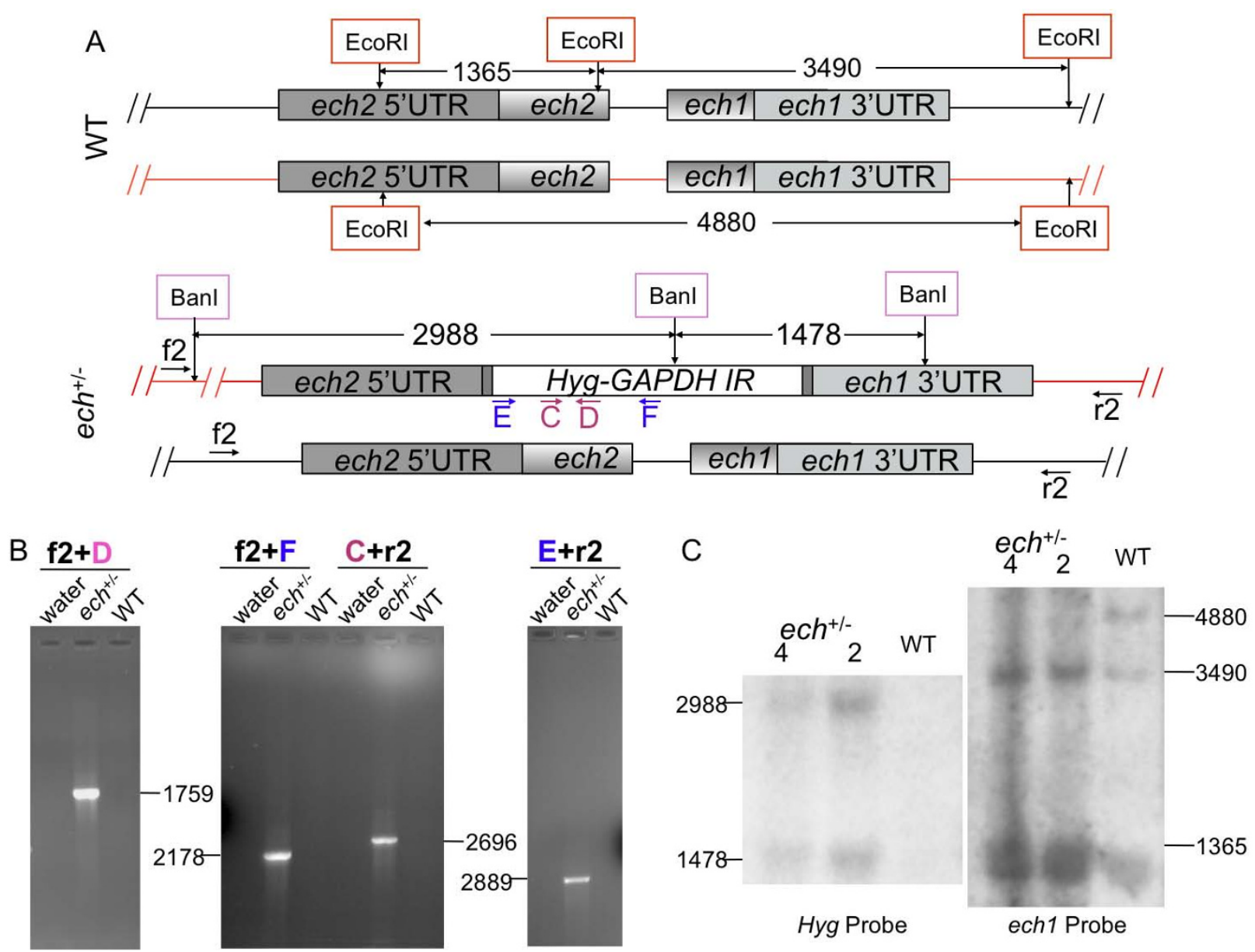

\section{Figure 3}

Simultaneous replacement of consecutive ech I and ech2 genes by a MS/GW construct pDEST/ech-Hyg-

GAPDH. A) Diagram of ech I, ech2 and Hyg-GAPDH-IR genomic loci in WT and ech ${ }^{+/-/ H y g}$ parasites. B) PCR genotyping analysis of: no template control (water); ech ${ }^{+/-} / \mathrm{Hyg}\left(\mathrm{ech}^{+/}\right)$and WT CL (WT). See additional file 3: Table S5 for nucleotide sequences of primers. C) Southern blot analysis of two clones ( 2 and 4$)$ of ech ${ }^{+/-} / \mathrm{Hyg}$. Left panel, gDNA digested with Banl and hybridized with Hyg CDS; right panel, gDNA digested with EcoRI and hybridized with labeled ech I CDS. Diagrams not to scale. Numbers are sizes (bp) of expected products.

showed a 3884 bp Neo gene band in the $e \mathrm{ch}^{+/} / \mathrm{Neo}$ parasites (Figure 4C).

\section{One-step-PCR knockout strategy fails to delete dhfr-ts and ech genes}

Since we demonstrated that at least one allele of the $d h f r$ ts can be deleted using the MS/GW based system, we next tested if this gene can be deleted using the one-step-PCR strategy. Transfection and selection of parasites with the knockout cassette LP-dhfr-ts-Neo failed to yield drug resistant parasites, despite 4 independent attempts. As there are $78 \mathrm{nts}$ of the CDS of $d h f r-t s$ gene in both forward long primers used to produce LP-dhfr-ts-Neo, the drug selectable markers were to be expressed as a fusion pro- tein, with 26 amino acids of the start of $d h f r-t s$ gene fused at the $\mathrm{N}$ terminal. It is possible that the knockout parasites were not obtained because the drug selectable marker has reduced enzyme activity when expressed as a fusion protein. To exclude this possibility, we constructed LP-dhfr-tsUTR-Neo to completely delete the entire $d h f r$-ts sequence. This construct has $78 \mathrm{nts}$ of the UTR of $d h f r-t s$ gene instead of the CDS, providing production of neomycin phosphotransferase as a non-fusion protein. However, as with the previous construction, no resistant parasites could be obtained despite 4 independent electroporations. Furthermore, one-step-PCR strategy also failed to delete the ech1 and ech 2 genes despite 5 independent transfection and selection attempts. Therefore, the constructs generated 

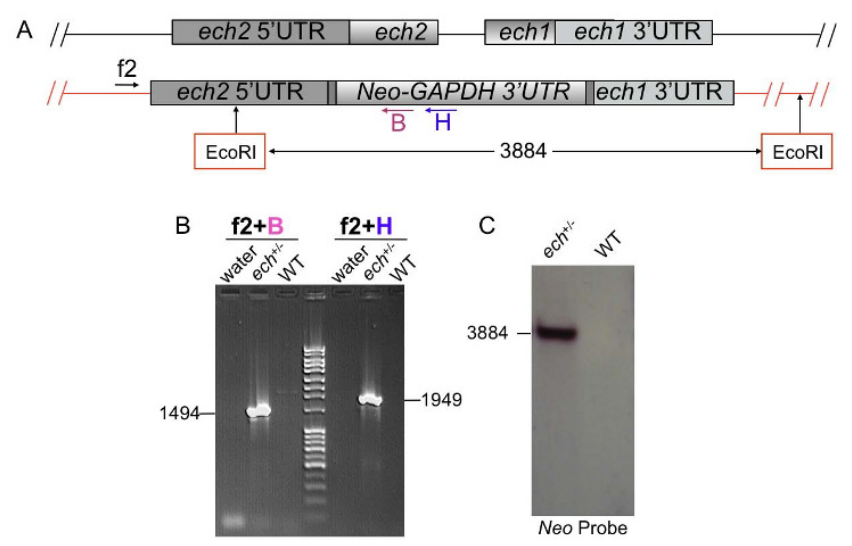

\section{Figure 4}

Simultaneous replacement of consecutive ech $I$ and ech2 genes by another MS/GW construct PDEST/ ech_Neo-GAPDH. A) Diagram of ech I, ech2 and NeoGAPDH 3'UTR genomic loci in ech ${ }^{+/} /$Neo parasites. B) PCR genotyping analysis of: no template control (water); $\mathrm{ech}^{+1-1}$ Neo $\left(\mathrm{ech}^{+/}\right)$and WT CL (WT). See additional file 3: Table S5 for nucleotide sequences of primers. C) Southern blot analysis of WT CL (WT) and ech $/$ / $/ \mathrm{Neo}\left(\mathrm{ech}^{+/}\right)$digested with EcoRI and hybridized with Neo CDS. Diagram not to scale. Numbers are sizes (bp) of expected products.

with one-step-PCR strategy that bear $78 \mathrm{nts}$ gene CDS or UTR specific sequence are likely to be insufficient for homologous recombination in T. cruzi.

\section{Discussion}

Experimental characterization of gene functions in trypanosomatids has relied heavily on reverse genetic approaches and has been facilitated by the development and optimization of gene manipulation strategies and transfection protocols [30]. In contrast to the robust and extensive techniques for genetic manipulation documented in Trypanosoma brucei and Leishmania, the validated techniques and record of success for $T$. cruzi is much less extensive. A goal of this study was to validate gene $\mathrm{KO}$ strategies for $T$. cruzi which might facilitate research on this important cause of human disease.

Toward that end, we have compared a conventional multi-step cloning technique with two knockout strategies that have been proven to target gene deletion in other organisms, one-step-PCR and MultiSite Gateway. The appeal of the one-step-PCR- strategy is the speed with which constructs can be produced. However, the attempts to knockout either ech or $d h f r$-ts genes in T. cruzi using this approach were unsuccessful, presumably because the 78 nucleotide-gene-specific regions used in our constructs were insufficient for homologous recombination in $T$. cruzi. This result is perhaps not surprising as studies in Leishmania [32] demonstrated that at least 150 nucle- otides are needed to guide homologous recombination. However, a recombination rate of $4 \times 10^{-4}$ was obtained with as short as 42 nucleotides homology in T. brucei [33]. Because of the considerable expense of oligos of $>100 \mathrm{bp}$, we did not investigate the minimum length needed for consistent recombination in T. cruzi, believing such an approach to be impractical for economical, high-efficiency gene knockouts.

The MultiSite Gateway-based approach, although not as simple as the one-step-PCR strategy, is far less time-consuming than the standard conventional methods. In particular, extensive restriction mapping, digestion and ligation steps are not needed at all with the MS/GW approach [34]. pDONR vectors containing drug resistance genes can be generated once and then repeatedly re-used for production of knockout constructs for different genes, further increasing the efficiency of the process. Once regions flanking the genes of interest are obtained from the att- PCR amplifications, the knockout DNA constructs can be generated within as few as five days (Figure 5). The $\mathrm{BP}$ and LR reactions are robust and have very high success rates; typically, at least $90 \%$ colonies screened from our $\mathrm{BP}$ and LR reactions are positive. Using the MS/GW knockout constructs, we successfully obtained $d h f r-t s^{+/}$ and $e \mathrm{ch}^{+/}$- parasites in two different $T$. cruzi strains. In ongoing work, we have used MS/GW constructs to successfully produce single as well as double KO lines for more than 10 other genes, ranging in size from 828 to 2730 nucleotides and up to 3 copies (using additional drug resistance markers). Thus the MS/GW approach appears to be amenable to use as part of a higher throughput gene knockout project.

Overall, the results described here identify the Multisite Gateway (MS/GW) -based system as an efficient tool to create knockout construction for deletion of genes in $T$. cruzi and should help accelerate the functional analysis of a wider array of genes in this important agent of disease.

\section{Conclusion}

This study documents the development of a Multisite Gateway based method for efficient gene knockout in $T$. cruzi. Further, we demonstrate that long-primer-based KO constructs with $<80$ nucleotides of homologous gene sequences are insufficient for consistent homologous recombination in $T$. cruzi. The increase in efficiency of gene knockout constructs should facilitate increased throughput for the identification of gene function in $T$. cruzi using reverse genetics.

\section{Methods \\ Culture, transfection and cloning of T. cruzi}

CL and Tulahuen lines of T. cruzi epimastigotes were cultured at $26^{\circ} \mathrm{C}$ in supplemented liver digest-neutralized 
tryptose (LDNT) medium as described previously [35]. A total of $1 \times 10^{7}$ early-log epimastigotes were centrifuged at $1,620 \mathrm{~g}$ for $15 \mathrm{~min}$ and resuspended in $100 \mu \mathrm{l}$ room temperature Human T Cell Nucleofector ${ }^{\mathrm{TM}}$ Solution (Amaxa AG, Cologne, Germany). The resuspended parasites were then mixed with 3-10 $\mu$ g DA (8-10 $\mu \mathrm{g}$ DNA for constructs generated using the conventional and MS/GW strategy; 3-10 $\mu \mathrm{g}$ DNA for constructs generated through one-step-PCR) in a total volume of 5-10 $\mu$ l and electroporated using program "U-33" in an AMAXA Nucleofector Device. This protocol generally yields $6-13 \%$ yellow fluorescent protein (YFP) positive parasites 24 hrs after transfection using $10 \mu \mathrm{g}$ of a YFP-containing control plasmid. The electroporated parasites are cultured in $25 \mathrm{~cm}^{2}$ cell culture flasks (Corning Incorporated, Lowell, MA, USA) with $10 \mathrm{ml}$ LDNT medium; $250 \mu \mathrm{g} / \mathrm{ml} \mathrm{G418} \mathrm{(for} \mathrm{trans-}$ fectants with neomycin phosphotransferase gene-containing cassette) and/or $600 \mu \mathrm{g} / \mathrm{ml}$ Hyg (Hygromycin B, for transfectants with hygromycin reisitance gene-containing cassette) was added at 24 hrs post-transfection. Parasites were considered fully selected when parasites transfected with no DNA were dead, generally at 4-5 weeks posttransfection. For single-cell cloning, drug selected lines were deposited into a 96-well plate to a density of 1 cell/ well using a MoFlow (Dako-Cytomation, Denmark) cell sorter and cultured in $250 \mu \mathrm{l}$ LDNT supplemented with G418 or Hyg. Each population from an individual well was considered an individual clone.
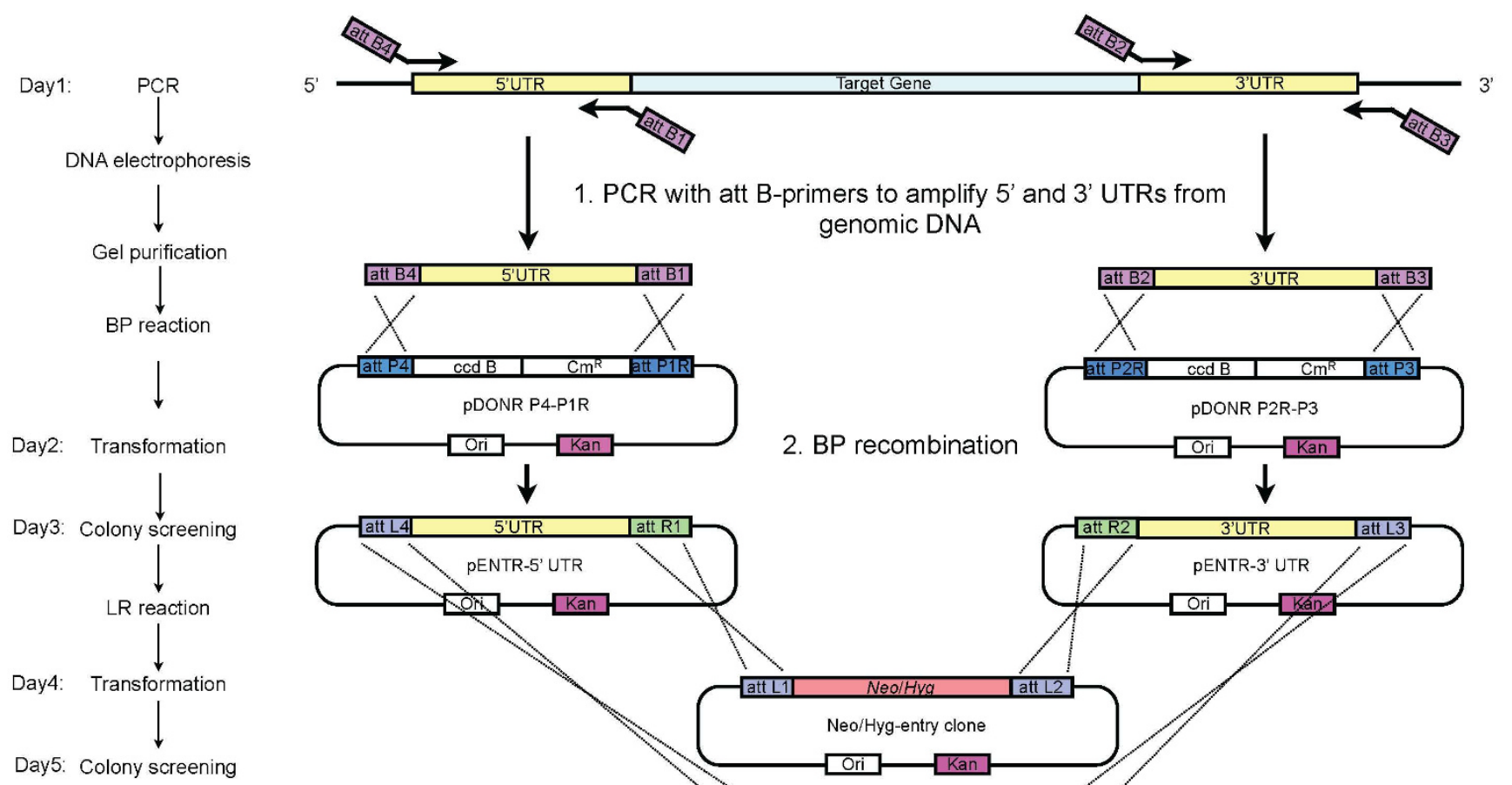

2. BP recombination
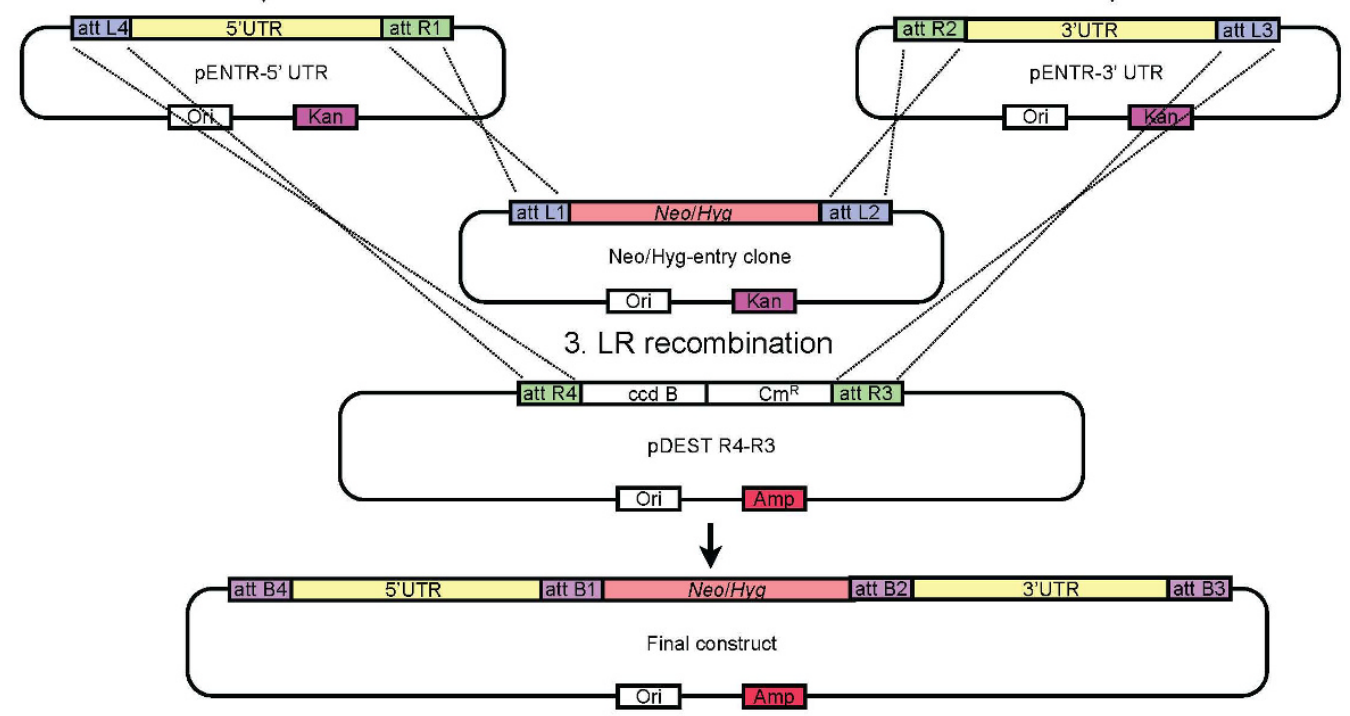

\section{Figure 5}

Timeline for constructing a KO plasmids using MS/GW strategy. The Multisite Gateway based method consists of three steps: I) PCR with attB-containing primers to amplify 5' and 3' UTR from genomic DNA; 2) BP recombination of each PCR products with specific donor vectors to generate entry clones containing the UTRs; 3) LR recombination of the two entry clones made in step 2 and a third entry clone containing Neo/Hyg to create the final construct. (Kan, kanamycin-resistance gene; Amp, ampicillin-resistance gene; Ori, Origin of replication). 


\section{Construction of a knockout DNA cassette using the conventional strategy}

The complete coding sequence of $1566 \mathrm{bp}$ of the dhfr-ts gene was amplified by PCR from genomic DNA (gDNA) of the WT Tulahuen strain using AmpliTaq Gold ${ }^{\circledR}$ DNA Polymerase (Roche) and primers DH5_f and DH6_r (Additional file 5: Table S1). The PCR product was gel purified with QIAquick Gel Extraction Kit (Qiagen, Valencia, CA, USA), treated with T4 DNA Polymerase (BioLabs) to generate blunt ends and cloned into the KpnI-digested, T4 DNA Polymerase (BioLabs) treated and dephosphorylated pBlueScript SKII + (Stratagene). Then the dhfr-ts coding region was disrupted by inserting into the PshAI restriction site of the $d h f r$-ts gene the neomycin phosphotransferase gene which have been generated by digestion with NotI/StuI of pBSSK-neo1f8 plasmid [27]. The resulting recombination vector were sequenced and designated as pBSdh1f8Neo (Additional file 1: Figure S1) containing the Neo CDS plus the trans-splicing $1 \mathrm{f} 8$ region, as well as $1016 \mathrm{bp}$ and $550 \mathrm{bp}$ of the $5^{\prime}$ and 3 'dhfr-ts coding regions.

The final plasmid was digested with restriction enzyme KpnI to liberate the knockout DNA cassette, gel eluted, ethanol precipitated and resuspended in water to a final concentration of $1-2 \mu \mathrm{g} / \mu \mathrm{l}$.

\section{Construction of knockout DNA cassettes based on MS/GW strategy}

All plasmids were constructed based on MS/GW system using commercially available MultiSite Gateway ThreeFragment Vector Construction kit (Invitrogen, Carlsbad, CA, USA), which includes all the Entry vectors and Destination vectors used in this study (Figure 5). In the Gateway system, the "BP" reaction is a recombination reaction between attB and attP sites in the PCR fragment and Donor vectors, resulting in Entry clones contains the gene of interest flanked by attL sites. "LR" reactions allow recombination between attL and attR sites of a Destination vector to yield an expression clone.

\section{pDEST/dhfr-ts_IF8Hyg}

In order to construct the pDEST/dhfr-ts_1F8Hyg plasmid, 1.0-kb 5' flanking sequence of $d h f r$-ts was amplified from gDNA of the WT CL strain using primers attB4_5'UTR_dhfr_f and attB1_5'UTR_dhfr_r (Additional file 6: Table S2) and Platinum ${ }^{\circledR}$ PCR SuperMix (Invitrogen), gel purified with QIAquick Gel Extraction Kit (Qiagen, Valencia, CA, USA) and cloned into the Entry vector $\mathrm{pDONR}^{\mathrm{TM}} \mathrm{P} 4-\mathrm{P} 1 \mathrm{R}$ through a $\mathrm{BP}$ reaction using the $\mathrm{BP}$ Clonase II Enzyme Mix (Invitrogen), resulting in the Entry clone pDONR_5'UTR_dhfr. Similarly, 1.0-kb 3' flanking sequence of $d h f r$-ts was amplified using primers attB2_3'UTR_dhfr_f and attB3_3'UTR_dhfr_r (Additional file 6: Table S2) and cloned into $\mathrm{pDONR}{ }^{\mathrm{TM}} \mathrm{P} 2 \mathrm{R}-\mathrm{P} 3$ to generate pDONR_3'UTR_dhfr. Using plasmid pBSSK-hyg1f8 [27] as a template, the $H y g$ and its upstream $1 \mathrm{f} 8$ region was amplified with primers attB1_1F8_f and attB2_1F8Hyg_r (Additional file 6: Table S2) and cloned into Entry vector pDONR ${ }^{\mathrm{TM}} 221$. The three Entry clones were then mixed with a Destination vector pDEST $^{\mathrm{TM}} \mathrm{R} 4-\mathrm{R} 3$ in an LR reaction using the LR Clonase II Plus Enzyme Mix (Invitrogen) to generate a final plasmid pDEST/dhfrts_1F8Hyg (Additional file 2: Figure S2). The knockout DNA cassette was liberated from the plasmid backbone with AlwNI and PvuI enzymes, and purified as above.

\section{pDESTlech_Neo-GAPDH and pDEST/ech_Hyg-GAPDH}

Trypanosoma cruzi ech1 and ech2 are tandemly arranged genes. To construct the pDEST/ech_Hyg-GAPDH plasmid, $1.0-\mathrm{kb} 5$ ' sequence of ech2 was amplified with primers attB4_ech5'UTR_f and attB1_ech5'UTR_r (Additional file 6: Table S2), gel purified and cloned into the Entry clone pDONR-ech5'UTR. Similarly, 1.0-kb 3' sequence of ech1 was amplified with primers attB2_ech3'UTR_f and attB3_ech3'UTR_r (Additional file 6: Table S2) and cloned into pDONR ${ }^{\mathrm{TM}} \mathrm{P} 2 \mathrm{R}-\mathrm{P} 3$ to generate pDONR-ech3'UTR. $H y g$ and the downstream intergenic region of GAPDH (glyceraldehyde-3-phosphate dehydrogenase) (GAPDH-IR) was amplified from plasmid pTEX-Hyg.mcs [36] using primers attB1_Hyg_f and attB2_Hyg_r (Additional file 6: Table S2) and cloned into Entry vector $\mathrm{pDONR}^{\mathrm{TM}} 221$. The three Entry clones were then mixed with a Destination vector pDEST $^{\text {TM }}$ R4-R3 to generate pDEST/ech_Hyg-GAPDH (Additional file 4: Figure $\mathrm{S} 3 \mathrm{~A}$ ) through a $\mathrm{LR}$ reaction. The final plasmid was digested with restriction enzymes PvulI and Pcil and purified as above.

Similarly, to construct pDEST/ech_Neo-GAPDH (Additional file 4: Figure S3B), Neo and 3'UTR of GAPDH (GAPDH 3'UTR) was amplified from plasmid pTrex-YFP (modified from the backbone of pTrex [37]) with primers attB1_Neo_f and attB2_Neo_r (Additional file 6: Table S2) and cloned into Entry vector $\mathrm{pDONR}^{\mathrm{TM}} 221$. The final plasmid was digested with restriction enzymes Pvul and Pcil and purified as above.

\section{Construction of knockout DNA cassettes via one-step-PCR} For the constructs for deletion of the dhfr-ts gene using one-step-PCR, Neo and Hyg was amplified with primers LP_dhfr_Neo_f and LP_dhfr_Neo_r, and LP_dhfr_Hyg_f and LP_dhfr_Hyg_r (Additional file 7: Table S3) from plasmids pTrex-YFP and pTEX-Hyg.mcs respectively. In both cases, forward primers and reverse primers corresponded to the $78 \mathrm{bp}$ downstream of the start codon of the $d h f r$-ts gene and reverse 78 bp upstream of the stop codon of the gene, respectively. 
In addition, primers LP_dhfr-UTR_Neo_f and LP_dhfrUTR_Neo_r, (Additional file 7: Table S3) were also used to amplify Neo from pTrex-YFP. In this case, LP_dhfrUTR_Neo_f included 78 bp upstream of the start codon of the dhfr-ts gene whereas LP_dhfr-UTR_Neo_r bears 78 bp downstream of the stop codon.

Likewise, primers LP_ech_Neo_f and LP_ech_Neo_r (Additional file 7: Table S3) were designed to amplify the final construction for deletion of the ech genes as well as primers LP_ech_Hyg_f and LP_ech_Hyg_r (Additional file 7: Table S3). PCR reactions were carried out as follows: initial denaturation at $94^{\circ} \mathrm{C}$ for $3 \mathrm{~min}$ followed by 30 cycles of: $98^{\circ} \mathrm{C}$ for $20 \mathrm{~s} ; 55^{\circ} \mathrm{C}$ for $30 \mathrm{~s}$; and $72^{\circ} \mathrm{C}$ for $2 \mathrm{~min}$ followed by $72^{\circ} \mathrm{C}$ for $10 \mathrm{~min}$ using Gradient Master Thermocycler (Eppendorf, Westbury, NY, USA). Products were collected and purified with QIAquick PCR Purification Kit. The eluted DNA was further ethanol precipitated and resuspended to $0.2-1 \mu \mathrm{g} / \mu \mathrm{l}$.

\section{Southern blot}

For Southern blot analysis, gDNA from different clones and strains was purified using Wizard Genomic DNA Purification Kit (Promega Corporation, Madison, WI, USA). The DNA was digested and separated by $0.7 \%$ agarose gel electrophoresis, and the gels blotted onto nylon membranes (Hybond-N 0.45-mm-pore-size filters; Amersham Life Science) using standard methods [38]. For probe generation, a 1030 bp DNA ( $H y g)$ was amplified using primers Hyg_f and Hyg_r (Additional file 8: Table S4) from plasmid pTEX-Hyg.mcs. For the Neo probe, a 795 bp DNA fragment was amplified from plasmid pBSSK-neo1f8 using primers Neo_f and Neo_r (Additional file 8: Table S4). ech1 gene were amplified using primers ech1_pb_f and ech1_pb_r (Additional file 8: Table S4) from gDNA of WT CL, while dhfr-ts gene was amplified from gDNA of WT Tulahuen using primers DH5_f and DH6_r (Additional file 5: Table S1). The PCR products were purified as above. Labeling of the probe and DNA hybridization were performed according to the protocol supplied with the PCR-DIG DNA-labeling and detection kit (Roche Applied Science, Indianapolis, IN, USA).

\section{Authors' contributions}

DX participated in the design of the study, carried out the ech gene knockout experiments, and drafted the manuscript. CPB participated in the design of the study, carried out the experiments to knockout the dhfr-ts gene, and revised this manuscript intensively. MAB participated in its design and coordination and revised the manuscript critically. RLT conceived of the study, participated in its design and coordination and revised the manuscript critically. All authors read and approved the final manuscript.

\section{Additional material}

\author{
Additional File 1 \\ Figure S1. Plasmid map of pBSdh1 f8Neo for conventional disruption of \\ the dhfr-ts gene. \\ Click here for file \\ [http://www.biomedcentral.com/content/supplementary/1471- \\ 2180-9-90-S1.pdf]
}

\section{Additional File 2}

Figure S2. Plasmid map of pDEST/dhfr-ts_1F8Hyg obtained by the MS/ GW system used for the deletion of the dhfr-ts gene.

Click here for file

[http://www.biomedcentral.com/content/supplementary/1471-

2180-9-90-S2.pdf]

\section{Additional File 3}

Table S5. Oligonucleotides for PCR analysis.

Click here for file

[http://www.biomedcentral.com/content/supplementary/1471-

2180-9-90-S3.doc]

\section{Additional File 4}

Figure S3. Maps of the plasmids obtained by the MS/GW system used for the deletion of the ech gene. A) pDEST/ech_Hyg-GAPDH and B) pDEST/ech_Neo-GAPDH.

Click here for file

[http://www.biomedcentral.com/content/supplementary/1471-

2180-9-90-S4.tiff]

\section{Additional File 5}

Table S1. Oligonucleotides for generation of knockout constructs based on the conventional strategy.

Click here for file

[http://www.biomedcentral.com/content/supplementary/1471-

2180-9-90-S5.doc]

\section{Additional File 6}

Table S2. Oligonucleotides for generation of knockout constructs based on the MS/GW strategy.

Click here for file

[http://www.biomedcentral.com/content/supplementary/1471-

2180-9-90-S6.doc]

\section{Additional File 7}

Table S3. Oligonucleotides for one-step-PCR.

Click here for file

[http://www.biomedcentral.com/content/supplementary/1471-

2180-9-90-S7.doc]

\section{Additional File 8}

Table S4. Oligonucleotides for probe generation of Southern blot analysis. Click here for file

[http://www.biomedcentral.com/content/supplementary/1471-

2180-9-90-S8.doc]

\section{Acknowledgements}

We are grateful to Dr. Angel M. Padilla and Dr. Todd Minning for valuable comments throughout this study. We would like to thanks Dr. Mirella Ciac- 
cio for her help in the initial steps of the work with the dhfr-ts gene, Dr. Antonio Gonzalez for facilitating the construction of the plasmids PBSSKneolf8 and pBSSK-hygIf8, Dr. Becky Bundy, Courtney Boehlke and Laura Simpson for their technical assistance, and Daniel B. Weatherly for bioinformatics expertise. This work was supported by NIH Grant POI Al0449790 to RLT.

\section{References}

I. Barrett MP, Burchmore RJ, Stich A, Lazzari JO, Frasch AC, Cazzulo J], Krishna S: The trypanosomiases. 362(9394): | 469-|480.

2. Control of Chagas disease. World Health Organ Tech Rep Ser 2002 905:i-iv. I-109, back cover

3. TDR/PAHO/WHO Scientific Working Group Report: Reporte sobre la enfermedad de Chagas. 2007 [http://www.who.int tdsvc/publications/tdr-research-publications/reporte-enfermedad-cha gas].

4. Tyler KM, Engman DM: The life cycle of Trypanosoma cruzi revisited. Int I Parasitol 200 I, 3 I (5-6):472-48I.

5. El-Sayed NM, Myler PJ, Bartholomeu DC, Nilsson D, Aggarwal G, Tran A-N, Ghedin E, Worthey EA, Delcher AL, Blandin G, et al: The Genome Sequence of Trypanosoma cruzi, Etiologic Agent of Chagas Disease. Science 2005, 309(5733):409-4I5.

6. Obado SO, Taylor MC, Wilkinson SR, Bromley EV, Kelly JM: Func tional mapping of a trypanosome centromere by chromosome fragmentation identifies a I6-kb GC-rich transcriptional "strand-switch" domain as a major feature. Genome Res 2005, I 5(I):36-43.

7. Machado CA, Ayala FJ: Nucleotide sequences provide evidence of genetic exchange among distantly related lineages of Trypanosoma cruzi. Proc Natl Acad Sci U S A 200I, 98(I3):7396-740I.

8. Cooper R, de Jesus AR, Cross GA: Deletion of an immunodominant Trypanosoma cruzi surface glycoprotein disrupts flagellum-cell adhesion. J Cell Biol I993, I 22(I): |49-I56.

9. Ajioka J, Swindle J: The calmodulin-ubiquitin (CUB) genes of Trypanosoma cruzi are essential for parasite viability. $\mathrm{Mol}$ Biochem Parasitol 1996, 78(I-2):217-225.

10. Caler EV, Vaena de Avalos S, Haynes PA, Andrews NW, Burleigh BA Oligopeptidase B-dependent signaling mediates host cell invasion by Trypanosoma cruzi. Embo J 1998 I 7( I 7):4975-4986.

11. Allaoui A, Francois C, Zemzoumi K, Guilvard E, Ouaissi A: Intracellular growth and metacyclogenesis defects in Trypanosoma cruzi carrying a targeted deletion of a Tc52 protein-encoding allele. Mol Microbiol 1999, 32(6): |273-1286.

12. Manning-Cela R, Cortes A, Gonzalez-Rey E, Van Voorhis WC, Swindle J, Gonzalez A: LYT I protein is required for efficient in vitro infection by Trypanosoma cruzi. Infect Immun 200I, 69(6):3916-3923.

13. Conte I, Labriola C, Cazzulo J], Docampo R, Parodi A): The inter play between folding-facilitating mechanisms in Trypanosoma cruzi endoplasmic reticulum. Mol Biol Cell 2003 , | 4(9):3529-3540.

14. Annoura T, Nara T, Makiuchi T, Hashimoto T, Aoki T: The origin of dihydroorotate dehydrogenase genes of kinetoplastids, with special reference to their biological significance and adaptation to anaerobic, parasitic conditions. I Mol Evol 2005, 60(I): I13-127.

I5. MacRae JI, Obado SO, Turnock DC, Roper JR, Kierans M, Kelly JM, Ferguson MAJ: The suppression of galactose metabolism in Trypanosoma cruzi epimastigotes causes changes in cell surface molecular architecture and cell morphology. Mol Biochem Parasitol 2006, I47(I): I26-136.

16. Barrio AB, Van Voorhis WC, Basombrl̀o MA: Trypanosoma cruzi: Attenuation of virulence and protective immunogenicity after monoallelic disruption of the cub gene. Experimental Parasitology 2007, I I 7(4):382-389.

17. Gluenz E, Taylor MC, Kelly JM: The Trypanosoma cruzi metacyclic-specific protein Met-III associates with the nucleolus and contains independent amino and carboxyl terminal targeting elements. Int J Parasitol 2007, 37(6):617-625.
18. Wilkinson SR, Taylor MC, Horn D, Kelly JM, Cheeseman I: A mechanism for cross-resistance to nifurtimox and benznidazole in trypanosomes. Proc Natl Acad Sci USA 2008, I 05( I 3):5022-5027.

19. Clayton CE: Genetic manipulation of kinetoplastida. Parasito Today 1999, I 5(9):372-378.

20. DaRocha WD, Otsu K, Teixeira SMR, Donelson JE: Tests of cytoplasmic RNA interference (RNAi) and construction of a tetracycline-inducible T7 promoter system in Trypanosoma cruzi. Mol Biochem Parasitol 2004, I33(2): I75-186.

21. Bellofatto V, Palenchar JB: RNA interference as a genetic tool in trypanosomes. Methods Mol Biol 2008, 442:83-94.

22. Reche P, Arrebola R, Olmo A, Santi DV, Gonzalez-Pacanowska D, Ruiz-Perez LM: Cloning and expression of the dihydrofolate reductase-thymidylate synthase gene from Trypanosoma cruzi. Mol Biochem Parasitol 1994, 65(2):247-258.

23. Reche P, Arrebola R, Santi DV, Gonzalez-Pacanowska D, Ruiz-Perez LM: Expression and characterization of the Trypanosoma cruzi dihydrofolate reductase domain. Mol Biochem Parasitol 1996, 76(I-2): I75-185.

24. Anderson AC: Targeting DHFR in parasitic protozoa. Drug Discov Today 2005, I 0(2): | $21-128$

25. Cruz A, Coburn CM, Beverley SM: Double targeted gene replacement for creating null mutants. Proc Natl Acad Sci USA |99|, 88(16):7|70-7|74.

26. Sienkiewicz N, Jaroslawski S, Wyllie S, Fairlamb AH: Chemical and genetic validation of dihydrofolate reductase-thymidylate synthase as a drug target in African trypanosomes. Mol Microbiol 2008, 69(2):520-533.

27. Thomas MC, Gonzalez A: A transformation vector for stagespecific expression of heterologous genes in Trypanosoma cruzi epimastigotes. Parasitol Res 1997, 83(2): | $5 \mid-156$.

28. Atwood JA 3rd, Weatherly DB, Minning TA, Bundy B, Cavola C Opperdoes FR, Orlando R, Tarleton RL: The Trypanosoma cruzi proteome. Science 2005, 309(5733):473-476.

29. Das A, Bellofatto V: Genetic regulation of protein synthesis in trypanosomes. Curr Mol Med 2004, 4(6):577-584.

30. Teixeira SM, daRocha WD: Control of gene expression and genetic manipulation in the Trypanosomatidae. Genet Mol Res 2003, 2(I): |48-I58.

3I. Nozaki T, Cross GA: Effects of 3' untranslated and intergenic regions on gene expression in Trypanosoma cruzi. Mol Biochem Parasitol 1995, 75( I):55-67.

32. Papadopoulou B, Dumas C: Parameters controlling the rate of gene targeting frequency in the protozoan parasite Leishmania. Nucleic Acids Res 1997, 25(2I):4278-4286.

33. Gaud A, Carrington M, Deshusses J, Schaller DR: Polymerase chain reaction-based gene disruption in Trypanosoma brucei. Mol Biochem Parasitol 1997, 87( I): I I3-II 5.

34. liizumi S, Nomura Y, So S, Uegaki K, Aoki K, Shibahara K, Adachi N, Koyama H: Simple one-week method to construct gene-targeting vectors: application to production of human knockout cell lines. BioTechniques 2006, 4 I (3):3 I I-3 I6.

35. Tyler KM, Engman DM: Flagellar elongation induced by glucose limitation is preadaptive for Trypanosoma cruzi differentiation. Cell Motil Cytoskeleton 2000, 46(4):269-278.

36. Kelly JM, Ward HM, Miles MA, Kendall G: A Shuttle Vector Which Facilitates the Expression of Transfected Genes in Trypanosoma-Cruzi and Leishmania. Nucleic Acids Research 1992, 20( I 5):3963-3969.

37. Lorenzi HA, Vazquez MP, Levin MJ: Integration of expression vectors into the ribosomal locus of Trypanosoma cruzi. Gene 2003, $310: 91-99$

38. Sambrook J, Russel DW: Molecular Cloning. A Laboratory Manual. Volume I. 3rd edition. Cold Spring Harbor Laboratory Press; 2001 . 